\title{
Case Report \\ Response of Paroxysmal Nocturnal Hemoglobinuria Clone with Aplastic Anemia to Rituximab
}

\author{
Radha Raghupathy ${ }^{1,2}$ and Olga Derman ${ }^{2}$ \\ ${ }^{1}$ Division of Hematology, Department of Medicine, Montefiore Medical Center, 111E 210th Street, Bronx, NY 10467, USA \\ ${ }^{2}$ Department of Oncology, Montefiore Medical Center, 111E 210th Street, Bronx, NY 10467, USA
}

Correspondence should be addressed to Radha Raghupathy, rradha80@gmail.com

Received 19 December 2011; Accepted 1 February 2012

Academic Editors: D. Galanakis, M. Nagasawa, and P. Tsirigotis

Copyright ( 92012 R. Raghupathy and O. Derman. This is an open access article distributed under the Creative Commons Attribution License, which permits unrestricted use, distribution, and reproduction in any medium, provided the original work is properly cited.

\begin{abstract}
Paroxysmal nocturnal hemoglobinuria is caused by expansion of a hematopoietic stem cell clone with an acquired somatic mutation in the PIG-A gene. This mutation aborts the synthesis and expression of the glycosylphosphatidylinositol anchor proteins CD55 and CD59 on the surface of blood cells, thereby making them more susceptible to complement-mediated damage. A spectrum of disorders occurs in PNH ranging from hemolytic anemia and thrombosis to myelodysplasia, aplastic anemia and, myeloid leukemias. Aplastic anemia is one of the most serious and life-threatening complications of $\mathrm{PNH}$, and a $\mathrm{PNH}$ clone is found in almost a third of the cases of aplastic anemia. While allogeneic bone marrow transplantation and $\mathrm{T}$ cell immune suppression are effective treatments for aplastic anemia in $\mathrm{PNH}$, these therapies have significant limitations. We report here the first case, to our knowledge, of PNH associated with aplastic anemia treated with the anti-CD20 monoclonal antibody rituximab, which was associated with a significant reduction in the size of the PNH clone and recovery of hematopoiesis. We suggest that this less toxic therapy may have a significant role to play in treatment of $\mathrm{PNH}$ associated with aplastic anemia.
\end{abstract}

\section{Introduction}

Severe aplastic anemia (SAA) is a bone marrow failure syndrome characterized by pancytopenia due to the absence of hematopoietic stem cells in the marrow. SAA is usually fatal, although spontaneous remissions have been documented. Most cases of SAA are idiopathic, but several triggers including drugs, radiation, viral infections, and chemical exposure have been described [1]. Activation of the T cell immune response by these inciting events and subsequent autoimmune destruction of hematopoietic stem cells appears to be pathogenic in most cases of SAA. This theory of T cellmediated autoimmunity in SAA is further supported by the therapeutic potential of $\mathrm{T}$ cell immune suppression in the disease [2].

A significant proportion of cases of AA, at some point in their evolution, are associated with a paroxysmal nocturnal hemoglobinuria (PNH) clone. PNH is a clonal hematopoietic disorder characterized by a somatic mutation in the PIGA gene that results in deficiency of complement protective glycosylphosphatidylinositol proteins CD55 and CD59 on the surface of blood cells. Complement-mediated intravascular hemolysis and thromboses are classical manifestations of $\mathrm{PNH}$ [3]. PNH can also evolve into other marrow disorders including AA, myelodysplasia, and acute myelogenous leukemia [4]. About 20 to $40 \%$ of patients with AA have a $\mathrm{PNH}$ clone at diagnosis. Late identification of a $\mathrm{PNH}$ clone following immunosuppressive therapy in AA has also been described [5-7]. Preferential survival of PNH clones in AA marrows has been attributed to relative resistance of the $\mathrm{PNH}$ clone to T cell cytotoxicity. Due to the decreased glycoprotein anchoring ability of the PNH cell, there is a decreased copy number of CD58 (lymphocyte function-associated antigen 3-ligand (LFA3)) on the red cell. Erythrocyte CD58 normally binds to $\mathrm{CD} 2$ and is responsible for $\mathrm{T}$ cell adhesion, activation, cytokine production and cytotoxic activity. CD58-depleted PNH cells are, therefore, relatively resistant to $\mathrm{T}$ cell-mediated effects, including autoimmunity $[8,9]$. On the other hand, PNH clones may be more sensitive to B-cell suppression by the monoclonal anti-CD20 antibody rituximab. Preclinical data show that upregulation of the GPI anchor proteins may mediate resistance to rituximab, and 
the absence of these surface proteins in $\mathrm{PNH}$ may make the clone more susceptible to rituximab cytotoxicity [10]. Our case, to our knowledge, is the first of its kind to demonstrate an effect of rituximab in AA with a $\mathrm{PNH}$ clone.

\section{Case Report}

A 17-year-old Hispanic female at 12 weeks of pregnancy was referred in March 2007 for severe pancytopenia. She had presented with fatigue and shortness of breath during a routine prenatal visit. Her past medical history was unremarkable except for a first trimester miscarriage one year prior. She was taking prenatal vitamins and denied chemical exposure, smoking or alcohol use, or a family history of blood disorders. On exam there was severe conjunctival pallor but no scleral icterus. No lymphadenopathy or hepatosplenomegaly was palpable. Petechiae were noted on both lower extremities.

Complete blood counts showed pancytopenia: WBC: $3.8 \times 10^{9} / \mathrm{L}$; ANC: $1.4 \times 10^{9} / \mathrm{L}$; nadir ANC: $0.1 \times 10^{9} / \mathrm{L} ; \mathrm{Hb}$ : $3.4 \mathrm{~g} / \mathrm{dL}$; platelets: $3 \times 10^{9} / \mathrm{L}$. Reticulocyte count was markedly low at $0.1 \%$ with an absolute reticulocyte count of $2.6 \times 10^{9} / \mathrm{L}$. Liver, renal function, and bilirubin were normal. Bone marrow biopsy showed an acellular marrow with 0 to $5 \%$ cellularity with marked reduction to absent trilineage hematopoiesis. Flow cytometry demonstrated $<1 \% \mathrm{CD}^{+} 4^{+}$ $\mathrm{CD} 117^{+}$hematopoietic precursors. Bone marrow cytogenetics showed a normal female karyotype of 46XX; fluorescent in situ hybridization studies were negative for deletion $5 q 31$, deletion 7q31, monosomy 5 and 7, trisomy 8 and $20 \mathrm{q} 12$ and p53 deletion. Serology revealed prior immunity to CMV, EBV, and parvovirus B-19. Hepatitis A, B, and C, HIV, and HTLV serologies were negative as was ANA and genetic testing for Fanconi's anemia. Serum B12 and folate levels were normal. SPEP and UPEP did not demonstrate a monoclonal protein. Flow cytometry for erythrocyte CD55 and CD59 was normal. A diagnosis of idiopathic very severe aplastic anemia (VSAA) was made. No sibling or unrelated donor could be identified as compatible for allogeneic bone marrow transplant (ABMT).

The patient's pregnancy was managed with transfusion support. At 32 weeks of gestation she presented in preterm labor with Escherichia coli sepsis. With prenatal steroids, antibiotics, and platelet support, she delivered a normal baby boy vaginally. In July 2007, 2 weeks after delivery, she received a course of intravenous equine antithymocyte globulin (hATG: $40 \mathrm{mg} / \mathrm{kg}$ daily for 4 days) and oral cyclosporine $12 \mathrm{mg} /$ $\mathrm{kg} / \mathrm{d}$ titrated to cyclosporine levels of 200 to $400 \mathrm{ng} / \mathrm{mL}$. Neutrophils remained less than $1.0 \times 10^{9} / \mathrm{L}$, and she remained transfusion dependent. A second cycle of h-ATG and cyclosporine was given in October 2007, which was complicated by seizures at therapeutic cyclosporine levels, and the cyclosporine was stopped. Three months later, she still remained transfusion dependent with neutrophils less than $1.0 \times 10^{9} / \mathrm{L}$.

In January 2008, a course of rituximab $375 \mathrm{mg} / \mathrm{m}^{2}$ was administered weekly for four weeks. In March 2008 she demonstrated partial recovery of neutrophils with a neutrophil count consistently between $1 \times 10^{9} / \mathrm{L}$ and $1.5 \times 10^{9} / \mathrm{L}$ while remaining transfusion dependent for platelets and red cells.

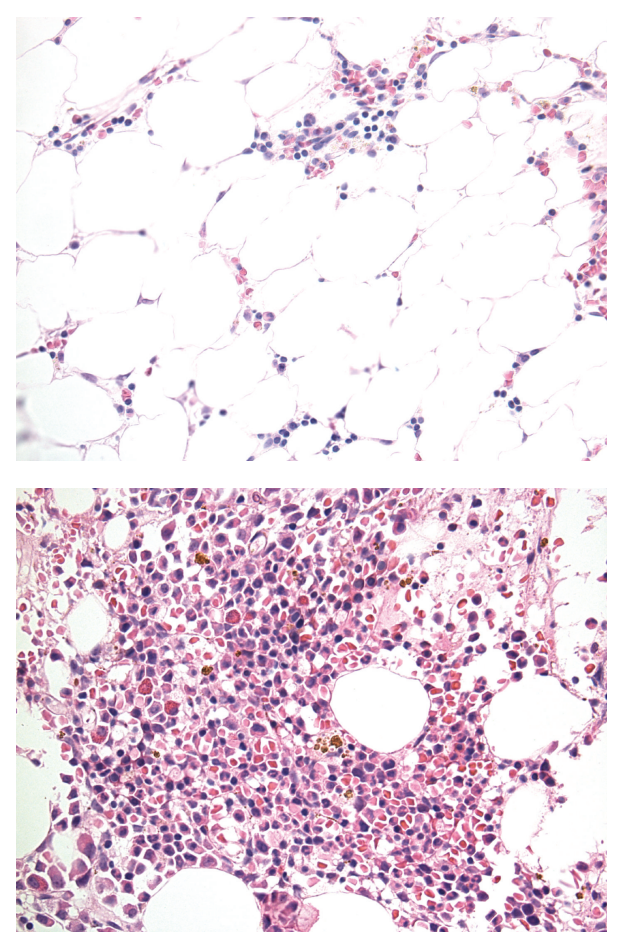

FIgURE 1: This figure illustrates the improvement in bone marrow biopsy findings with rituximab therapy. Pictures were taken under 40x magnification. The top figure shows the aplastic marrow on diagnosis in March 2007. The lower figure shows restoration of trilineage hematopoiesis with decreased megakaryocytes in July 2008, 6 months after the first cycle of rituximab.

At this time she was hospitalized with abdominal pain due to ischemic bowel and Budd-Chiari syndrome. Repeat testing for $\mathrm{PNH}$ was positive demonstrating CD59 deficiency on $81 \%$ of the leukocytes with $54.7 \%$ deficiency on the granulocytes. In addition, CD59 was found to be deficient on $11.4 \%$ of the red cells. Reticulocyte count was still low at $0.1 \%$ with absolute reticulocyte count of $2.6 \times 10^{9} / \mathrm{L}$. Lactate dehydrogenase was elevated at 245 Units/mL. Attempts to anticoagulate her were unsuccessful due to persistent bleeding despite platelet transfusions. She underwent bowel resection for ischemic bowel and was discharged after a prolonged and complicated hospital course. In May 2008, ANC stabilized between $1 \times 10^{9} / \mathrm{L}$ and $2 \times 10^{9} / \mathrm{L}$, but transfusion dependence for red cells and platelets persisted with nadir $\mathrm{Hb}$ of $5.5 \mathrm{gm} / \mathrm{dL}$ and platelets of $5 \times 10^{9} / \mathrm{L}$.

In July 2008, biweekly eculizumab was started with subsequent improvement in anemia and stabilization of hemoglobin at $9 \mathrm{gm} / \mathrm{dL}$ in May 2009. However platelet transfusions continued and neutrophils remained between 1 and $2 \times 10^{9} / \mathrm{L}$. Abdominal imaging showed resolution of the hepatic vein thromboses. No portosystemic collaterals, splenomegaly, or hepatic dysfunction had developed. Bone marrow biopsy showed a normocellular marrow (40\%) with erythroid predominant hematopoiesis and decreased megakaryocytes (Figure 1). While thrombocytopenia could be partly explained by slow megakaryocyte recovery, whether peripheral destruction by persistent autoimmune mechanisms also 


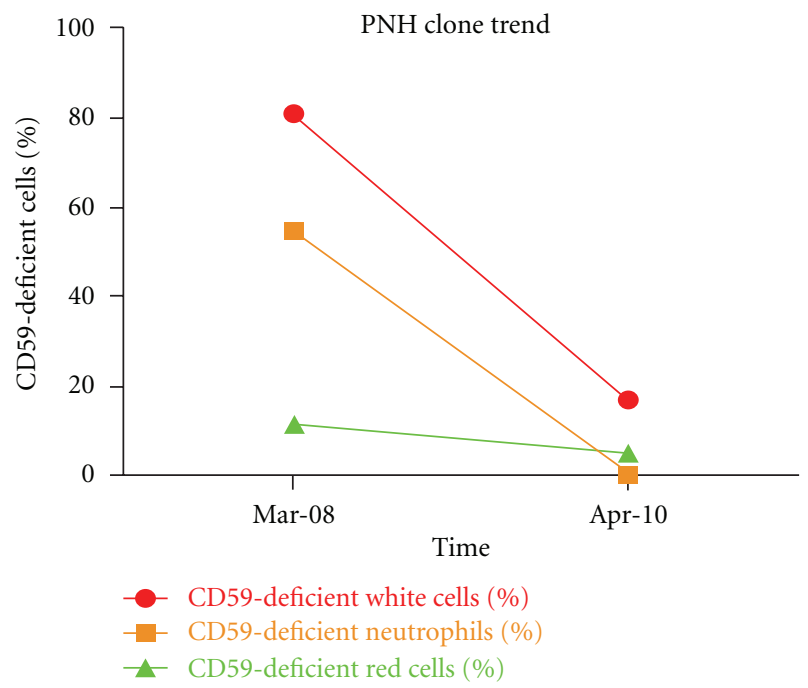

Figure 2: The reduction in $\mathrm{PNH}$ clone size over time. In March 2008, the CD59-deficient clone of WBCs and RBCs was detected when Budd-Chiari syndrome and intestinal ischemia were diagnosed. In April 2010, about 2 years after the first cycle of rituximab and 10 months after the second cycle of rituximab, there was significant decrease in size of the PNH clone in all cell lines.

contributed to ongoing pancytopenia is worth speculation. In June 2009, 17 months after the first cycle of rituximab, 4 additional doses of rituximab $375 \mathrm{mg} / \mathrm{m}^{2}$ weekly were given to facilitate platelet recovery. In October 2009, 4 months after the second course of rituximab, her hemoglobin normalized and her platelets began to recover. Repeat PNH testing in December 2009 and April 2010 showed significant decrease in the size of the PNH clone. In April 2010, only 17\% of the leukocytes, $0 \%$ of the granulocytes, and $5 \%$ of the red cells were deficient in CD59 (Trend of CD59, deficient cells in Figure 2). In November 2011, 2.5 years after the second course of rituximab, the patient remained in a complete hematological response ( $\mathrm{CBC}$ results shown in Figure 3 ).

\section{Discussion}

To our knowledge this is the first case report showing efficacy of rituximab in reducing PNH clone size and facilitating restoration of hematopoiesis in PNH associated with AA. The earliest response of improved hematopoiesis with rituximab in idiopathic AA was seen 50 days after therapy while complete response took up to 16 months [11]. In our case, partial neutrophil recovery was seen as early as two months after rituximab, hemoglobin response occurred about 1 year after therapy, and while the platelets did not recover with the first course of rituximab they showed dramatic recovery 3 months after the second course. The initial improvement in neutrophils in March 2008 could be partly attributed to the delayed effect of $\mathrm{T}$ cell suppression. Hemoglobin recovery may have been facilitated by eculizumab; however, anemia in this case was predominantly hypoproliferative rather than hemolytic suggesting a significant role of rituximab in its reversal. The improvement of megakaryopoiesis in October 2009 clearly had a temporal correlation with the second course of rituximab therapy.
In our case, testing for a PNH clone on erythrocytes was negative on presentation with AA. We propose that the $\mathrm{PNH}$ clone may have been undetected due to initial testing of predominantly transfused red cells and failure to test the white cells. The PNH clone in peripheral blood may have become manifest with improvement in neutrophil count. Alternatively $\mathrm{T}$ cell immune suppression may have facilitated emergence of the resistant PNH clone. While detection of the PNH clone occurred 2 months after rituximab therapy, we hypothesize this was not a direct rituximab effect. The complete effect of rituximab on B-cell immunity as well as possibly the PNH clone is likely to have taken several months. PNH clone size declined significantly between March 2008 and April 2010, temporally correlating with rituximab effect. Additional information like immunoglobulin levels and Bcell quantification may have further supported this argument but was not obtained at that time.

$\mathrm{PNH}$, an acquired hematopoietic stem cell disorder of the PIG-A gene, results in increased sensitivity of blood cells to complement-mediated destruction. Eculizumab, a humanized monoclonal antibody against $\mathrm{C} 5$, acts as a terminal complement inhibitor and is effective in targeting classical manifestations of PNH including complement-mediated hemolysis and thromboses [12]. However, eculizumab does not reduce PNH clone size or improve marrow failure in the disease [13]. ABMT is the only curative therapy for $\mathrm{PNH}$ that replaces the abnormal stem cell clone and improves hematopoiesis in concurrent AA. However, ABMT in PNH is limited by significant morbidity and mortality as well as donor availability, like in our case $[14,15]$.

$\mathrm{PNH}$ has a strong association with AA. Small to moderate PNH clones can be identified in a majority of patients with AA. In a retrospective analysis of 207 consecutive patients with severe $\mathrm{AA}, 40 \%$ of patients had a detectable PNH clone pretreatment and the median clone size was $9.7 \%$. In about $30 \%$ of these patients with a detectable PNH clone, the clone size increased after IST. Development of persistent new PNH clones after IST was rare and occurred only in $10 \%$ of patients. Classic PNH manifestations of hemolysis and thromboses in patients with AA and a PNH clone was uncommon and was seen only in 7 patients, all of whom had a PNH clone size of $>50 \%[16,17]$. Our patient demonstrates a rare case of a large PNH clone (81\% CD59-deficient leukocytes and $54.7 \%$ deficient granulocytes) evolving after IST for AA and presenting with a classic PNH manifestation of Budd-Chiari syndrome. Management in such cases needs to be directed against both the marrow aplasia and the PNH clone causing thrombotic manifestations.

Marrow failure in idiopathic AA and AA associated with $\mathrm{PNH}$ appears to be immune mediated. Activation of the $\mathrm{T}$ cell immune response and autoimmune destruction of hematopoietic stem cells appears to pathogenic in most cases of $\mathrm{AA}$ and therefore $\mathrm{T}$ cell suppression has emerged as effective therapy [2]. Normal T cells are activated by exposure to antigens on the surface of antigen-presenting cells. When activated, T cells produce IL-2, which results in further proliferation of their counterparts. Cytokines IFN- $\gamma$ and TNF- $\alpha$ are also produced by activated $\mathrm{T}$ cells, which result in upregulation of the Fas receptor causing apoptosis of target cells 


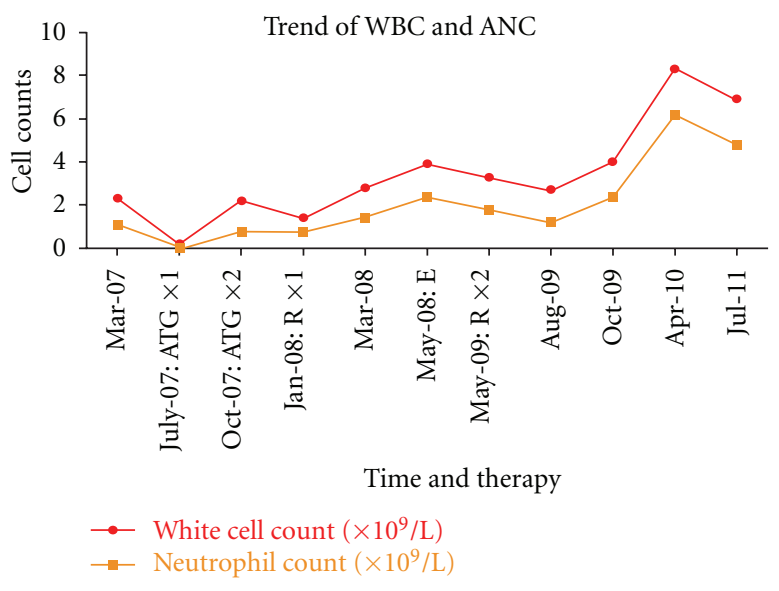

(a)

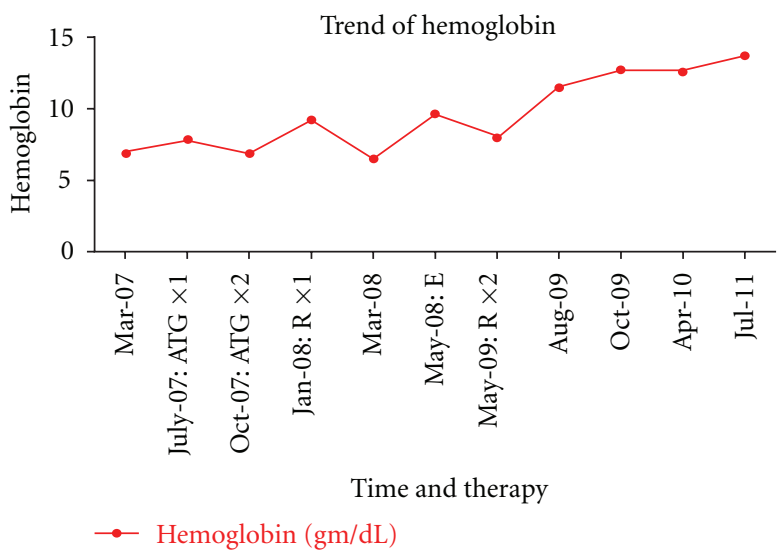

(b)

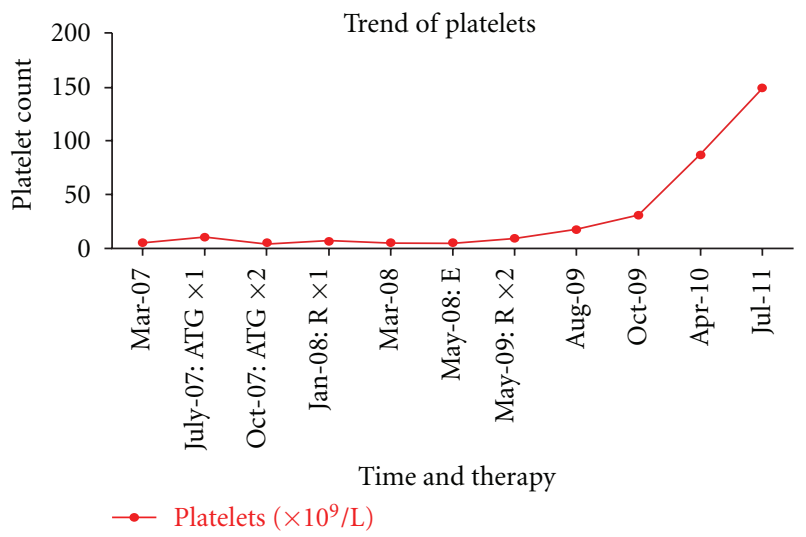

(c)

Figure 3: The improvement in blood counts from the time of diagnosis to most recent. (a) white cell and neutrophil counts, (b) describes the hemoglobin trend, and (c) the platelet trend. The $x$ axis is not drawn to scale and shows various time points and therapy administered. ATG: antithymocyte globulin, R: rituximab, and E: eculizumab.

[18]. In patients with $\mathrm{AA}$, often $\mathrm{T}$ cells are autoactivated resulting in excessive production of marrow toxic cytokines, IFN- $\gamma$, and TNF- $\alpha$. Many theories have been proposed for T cell autoactivation in AA. Tbet, a T cell transcription factor, appears to be constitutively active in T cells of patients with AA, stimulating production of IFN- $\gamma$ [19]. Polymorphisms in the cytokine encoding genes increasing synthesis of TNF$\alpha$, IFN- $\gamma$, and IL- 6 and causing apoptosis of marrow progenitors have also been described [20, 21]. Perforin gene mutations may cause abnormal proliferation and activation of cytotoxic T cells in AA [22]. T cell immune suppression is therapeutic in $75 \%$ of cases of AA [23]. However, PNH clones appear to be resistant to the $\mathrm{T}$ cell mediated cytotoxicity as well as $\mathrm{T}$ cell immune suppression facilitating preferential survival of the PNH clone in AA marrows. This phenomenon is demonstrated in our case $[16,24,25]$. Preferential survival of PNH clones after T cell suppression may be particularly problematic in situations where a large PNH clone causes classical PNH manifestations superimposed on marrow failure, as in our patient. Alternative therapeutic strategies are required in these cases and B-cell suppression can be explored.

B-cell autoimmunity and production of multiple autoantibodies have recently been described to be pathogenic in AA, especially the antibody to Moesin that has been shown to increase production of marrow toxic cytokines through activating the ERK 1/2 pathway [11]. Case reports have described the efficacy of rituximab in therapy of idiopathic AA $[11,26]$. Preclinical data also suggest that the absence of GPIlinked proteins may make the PNH clone exquisitely sensitive to rituximab-mediated cytotoxicity [10]. Our case report suggests that B-cell suppression by rituximab may be therapeutic for both marrow aplasia and the PNH clone.

AA is a uniformly fatal disease if left untreated, although rare cases of spontaneous remission have been described. Even with rapid institution of appropriate therapy, 10-year survival remains low. Median 10-year survival in AA is about $68 \%$ with immune suppressive therapy (IST) and $73 \%$ with ABMT [23]. More effective and less toxic therapies directed against the pathophysiology of AA are an urgent necessity 
to improve survival in this disease, especially since many afflicted patients are in their childhood and adolescence. In addition, management of a symptomatic large PNH clone in AA may be difficult, especially if a suitable ABMT donor is unavailable since the PNH clone is typically resistant to T cell immune suppression. Our case demonstrates that rituximab may be a very valuable drug with minimal toxicities in management of AA with a symptomatic large PNH clone. Larger clinical studies with correlative experiments will be required to validate our findings from this paper.

\section{Acknowledgments}

The authors would like to sincerely acknowledge Dr. Henny Billett and Dr. Roni Tamari for critically reviewing the paper and providing valuable suggestions. They would also like to thank Dr. Christine McMahon for her expert assistance in providing pictures of the bone marrow biopsy slides.

\section{References}

[1] N. S. Young, "Hematopoietic cell destruction by immune mechanisms in acquired aplastic anemia," Seminars in Hematology, vol. 37, no. 1, pp. 3-14, 2000.

[2] N. S. Young, "Pathophysiologic mechanisms in acquired aplastic anemia," Hematology/the Education Program of the American Society of Hematology, pp. 72-77, 2006.

[3] M. D. Cappellini, "Coagulation in the pathophysiology of hemolytic anemias," Hematology/the Education Program of the American Society of Hematology, pp. 74-78, 2007.

[4] P. Hillmen, S. M. Lewis, M. Bessler, L. Luzzatto, and J. V. Dacie, "Natural history of paroxysmal nocturnal hemoglobinuria," New England Journal of Medicine, vol. 333, no. 19, pp. 12531258, 1995.

[5] C. Parker, M. Omine, S. Richards et al., "Diagnosis and management of paroxysmal nocturnal hemoglobinuria," Blood, vol. 106, no. 12, pp. 3699-3709, 2005.

[6] D. E. Dunn, P. Tanawattanacharoen, P. Boccuni et al., "Paroxysmal nocturnal hemoglobinuria cells in patients with bone marrow failure syndromes," Annals of Internal Medicine, vol. 131, no. 6, pp. 401-408, 1999.

[7] N. S. Young, "Acquired aplastic anemia," Annals of Internal Medicine, vol. 136, no. 7, pp. 534-546, 2002.

[8] N. S. Young, "The problem of clonality in aplastic anemia: Dr Dameshek's riddle, restated," Blood, vol. 79, no. 6, pp. 13851392, 1992.

[9] T. Kinoshita and N. Inoue, "Relationship between aplastic anemia and paroxysmal nocturnal hemoglobinuria," International Journal of Hematology, vol. 75, no. 2, pp. 117-122, 2002.

[10] N. Nagajothi, W. H. Matsui, G. L. Mukhina, and R. A. Brodsky, "Enhanced cytotoxicity of rituximab following genetic and biochemical disruption of glycosylphosphatidylinositol anchored proteins," Leukemia and Lymphoma, vol. 45, no. 4, pp. 795-799, 2004.

[11] H. Takamatsu, H. Yagasaki, Y. Takahashi et al., "Aplastic anemia successfully treated with rituximab: the possible role of aplastic anemia-associated autoantibodies as a marker for response," European Journal of Haematology, vol. 86, no. 6, pp. 541-545, 2011.

[12] R. J. Kelly, A. Hill, L. M. Arnold et al., "Long-term treatment with eculizumab in paroxysmal nocturnal hemoglobinuria: sustained efficacy and improved survival," Blood, vol. 117, no. 25, pp. 6786-6792, 2011.

[13] P. Hillmen, N. S. Young, J. Schubert et al., "The complement inhibitor eculizumab in paroxysmal nocturnal hemoglobinuria," New England Journal of Medicine, vol. 355, no. 12, pp. 1233-1243, 2006.

[14] R. Saso, J. Marsh, L. Cevreska et al., "Bone marrow transplants for paroxysmal nocturnal haemoglobinuria," British Journal of Haematology, vol. 104, no. 2, pp. 392-396, 1999.

[15] R. A. Brodsky, "Stem cell transplantation for paroxysmal nocturnal hemoglobinuria," Haematologica, vol. 95, no. 6, pp. 855-856, 2010.

[16] P. Scheinberg, M. Marte, O. Nunez, and N. S. Young, "Paroxysmal nocturnal hemoglobinuria clones in severe aplastic anemia patients treated with horse anti-thymocyte globulin plus cyclosporine," Haematologica, vol. 95, no. 7, pp. 1075-1080, 2010.

[17] R. A. Brodsky, "Paroxysmal nocturnal hemoglobinuria: stem cells and clonality," Hematology/the Education Program of the American Society of Hematology, pp. 111-115, 2008.

[18] N. S. Young, R. T. Calado, and P. Scheinberg, "Current concepts in the pathophysiology and treatment of aplastic anemia," Blood, vol. 108, no. 8, pp. 2509-2519, 2006.

[19] E. E. Solomou, K. Keyvanfar, and N. S. Young, "T-bet, a Th1 transcription factor, is up-regulated in T cells from patients with aplastic anemia," Blood, vol. 107, no. 10, pp. 3983-3991, 2006.

[20] C. Dufour, M. Capasso, J. Svahn et al., "Homozygosis for (12) CA repeats in the first intron of the human IFN- $\gamma$ gene is significantly associated with the risk of aplastic anaemia in Caucasian population," British Journal of Haematology, vol. 126, no. 5, pp. 682-685, 2004.

[21] J. Demeter, G. Messer, and H. Schrezenmeier, "Clinical relevance of the TNF- $\alpha$ promoter/enhancer polymorphism in patients with aplastic anemia," Annals of Hematology, vol. 81, no. 10 , pp. 566-569, 2002.

[22] E. E. Solomou, F. Gibellini, B. Stewart et al., "Perforin gene mutations in patients with acquired aplastic anemia," Blood, vol. 109, no. 12, pp. 5234-5237, 2007.

[23] A. Locasciulli, R. Oneto, A. Bacigalupo et al., "Outcome of patients with acquired aplastic anemia given first line bone marrow transplantation or immunosuppressive treatment in the last decade: a report from the European Group for Blood and Marrow Transplantation (EBMT)," Haematologica, vol. 92, no. 1, pp. 11-18, 2007.

[24] A. Poggi, S. Negrini, M. R. Zocchi et al., "Patients with paroxysmal nocturnal hemoglobinuria have a high frequency of peripheral-blood $\mathrm{T}$ cells expressing activating isoforms of inhibiting superfamily receptors," Blood, vol. 106, no. 7, pp. 2399-2408, 2005.

[25] A. Karadimitris, J. S. Manavalan, H. T. Thaler et al., "Abnormal T-cell repertoire is consistent with immune process underlying the pathogenesis of paroxysmal nocturnal hemoglobinuria," Blood, vol. 96, no. 7, pp. 2613-2620, 2000.

[26] P. B. Hansen and A. M. F. Lauritzen, "Aplastic anemia successfully treated with rituximab," American Journal of Hematology, vol. 80, no. 4, pp. 292-294, 2005. 


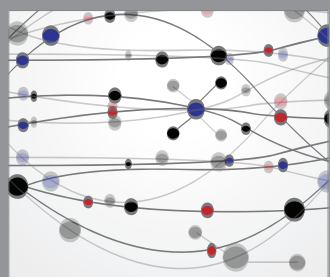

The Scientific World Journal
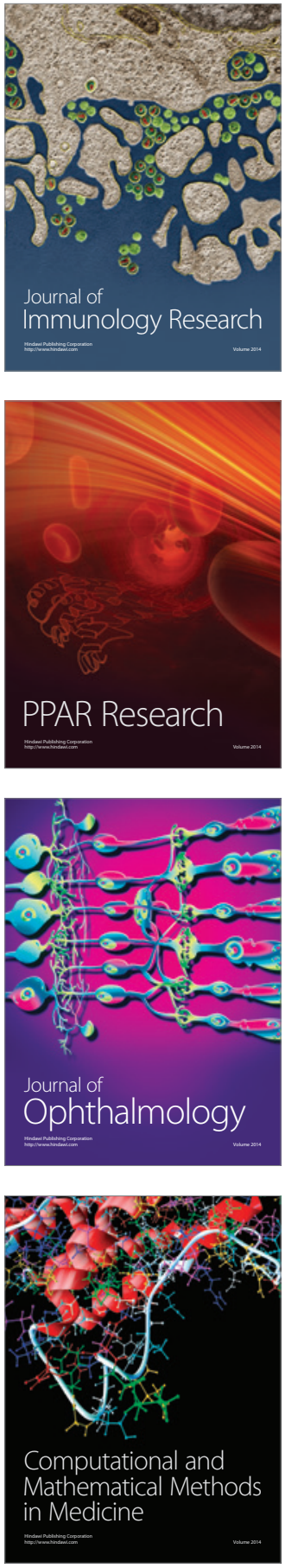

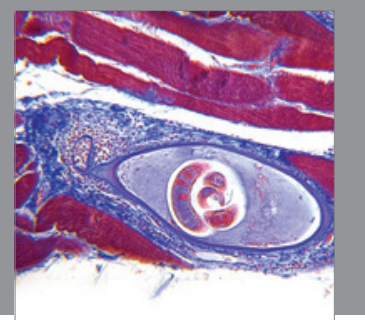

Gastroenterology

Research and Practice
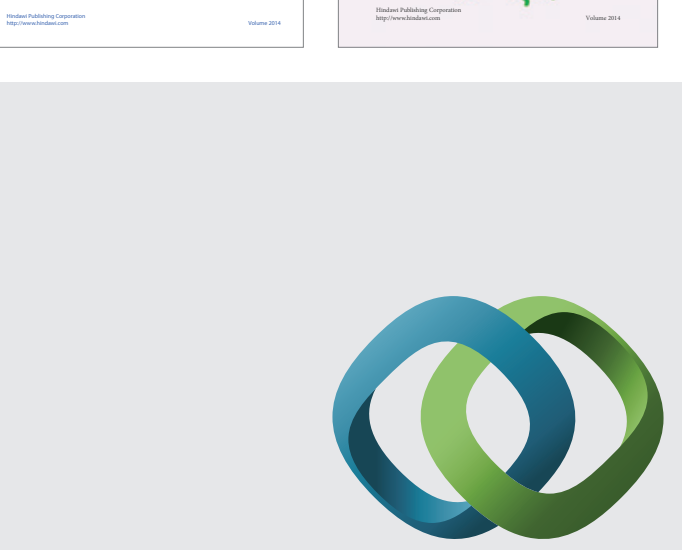

\section{Hindawi}

Submit your manuscripts at

http://www.hindawi.com
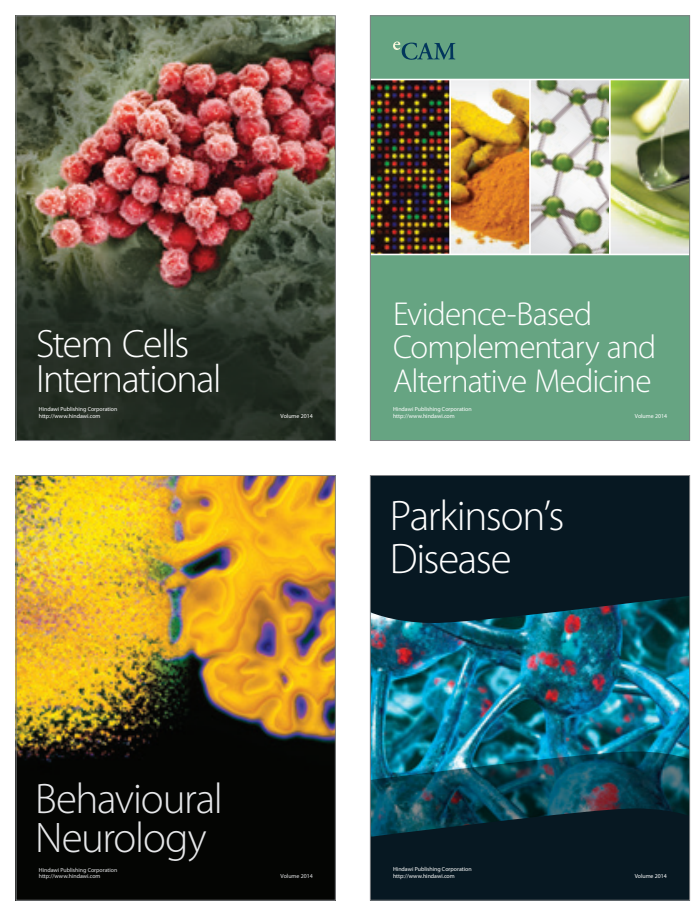

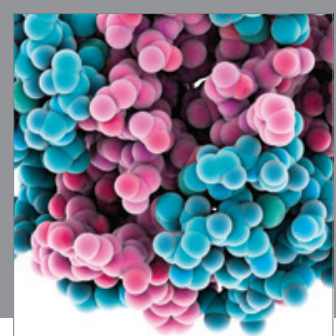

Journal of
Diabetes Research

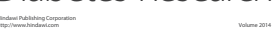

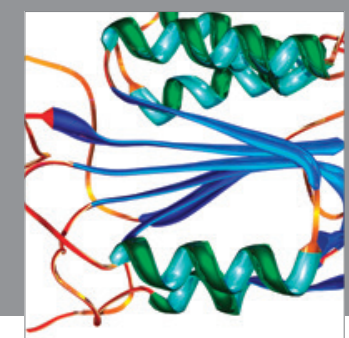

Disease Markers
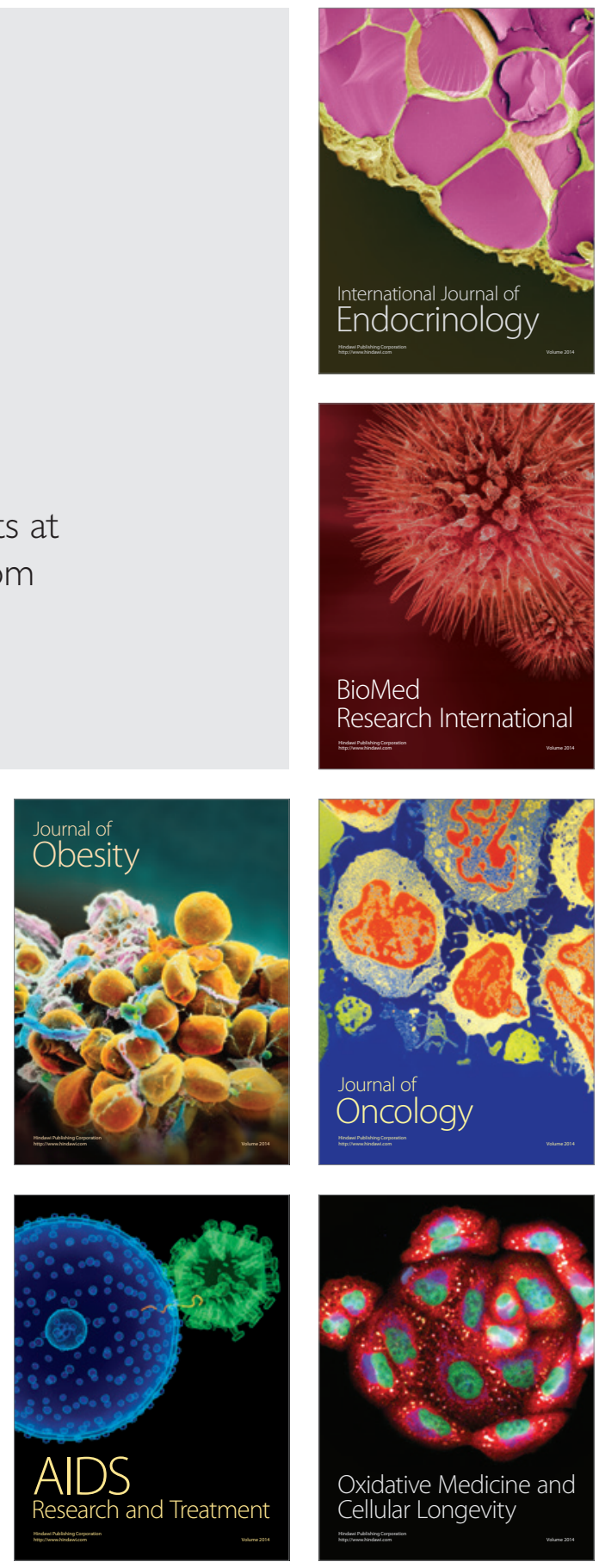